\title{
Escherichia coli Clonobiome: Assessing the Strain Diversity in Feces and Urine by Deep Amplicon Sequencing
}

\author{
(D) Sofiya G. Shevchenko, ${ }^{a}$ Matthew Radey, ${ }^{a}$ Veronika Tchesnokova, ${ }^{a}$ Dagmara Kisiela, ${ }^{a}$ Evgeni V. Sokurenko ${ }^{\text {* }}$ \\ aDepartment of Microbiology, University of Washington, Seattle, Washington, USA
}

ABSTRACT While microbiome studies have focused on diversity at the species level or higher, bacterial species in microbiomes are represented by different, often multiple, strains. These strains could be clonally and phenotypically very different, making assessment of strain content vital to a full understanding of microbiome function. This is especially important with respect to antibiotic-resistant strains, the clonal spread of which may be dependent on competition between them and susceptible strains from the same species. The pandemic, multidrug-resistant, and highly pathogenic Escherichia coli subclone ST131-H30 (H30) is of special interest, as it has already been found persisting in the gut and bladder in healthy people. In order to rapidly assess $E$. coli clonal diversity, we developed a novel method based on deep sequencing of two loci used for sequence typing, along with an algorithm for analysis of the resulting data. Using this method, we assessed fecal and urinary samples from healthy women carrying $\mathrm{H} 30$ and were able to uncover considerable diversity, including strains with frequencies at $<1 \%$ of the $E$. coli population. We also found that, even in the absence of antibiotic use, H3O could completely dominate the gut and, especially, urine of healthy carriers. Our study offers a novel tool for assessing a species' clonal diversity (clonobiome) within the microbiome, which could be useful in studying the population structure and dynamics of multidrug-resistant and/or highly pathogenic strains in their natural environments.

IMPORTANCE Bacterial species in the microbiome are often represented by multiple genetically and phenotypically different strains, making insight into subspecies diversity critical to a full understanding of the microbiome, especially with respect to opportunistic pathogens. However, methods allowing efficient high-throughput clonal typing are not currently available. This study combines a conventional E. coli typing method with deep amplicon sequencing to allow analysis of many samples concurrently. While our method was developed for E. coli, it may be adapted for other species, allowing microbiome researchers to assess clonal strain diversity in natural samples. Since assessment of subspecies diversity is particularly important for understanding the spread of antibiotic resistance, we applied our method to the study of a pandemic multidrug-resistant $E$. coli clone. The results we present suggest that this clone could be highly competitive in healthy carriers and that the mechanisms of colonization by such clones need to be studied.

KEYWORDS Escherichia coli, ST131, antibiotic resistance, bladder colonization, gut microbiome

M icrobiomes, in terms of both function and diversity, have recently been a topic of considerable interest. The gut microbiome has gotten special attention due to its high complexity and importance to health (1-9). So far, studies have almost exclusively focused on species or higher-level diversity. However, this paints an incomplete picture, since strains within the same species can be of distinct clonal origins and have vastly different metabolic, pathogenic, and antibiotic resistance profiles (10-19). Importantly,
Citation Shevchenko SG, Radey M, Tchesnokova V, Kisiela D, Sokurenko EV. 2019. Escherichia coli clonobiome: assessing the strain diversity in feces and urine by deep amplicon sequencing. Appl Environ Microbio 85:e01866-19. https://doi.org/10.1128/AEM .01866-19.

Editor Christopher A. Elkins, Centers for Disease Control and Prevention Copyright $\odot 2019$ American Society for Microbiology. All Rights Reserved.

Address correspondence to Evgeni V Sokurenko, evs@u.washington.edu.

* Present address: Evgeni V. Sokurenko, Department of Microbiology, University of Washington, Seattle, Washington, USA.

Received 15 August 2019

Accepted 12 September 2019

Accepted manuscript posted online 20 September 2019

Published 14 November 2019 
multidrug-resistant bacterial strains have been found competing with commensal strains in the gut, even without antibiotic pressure (18-23). Thus, there is a pressing need to identify strains in the human microbiome for species of critical health importance.

Escherichia coli is one of the most common residents of the gut. While primarily a commensal colonizer, extraintestinal pathogenic E. coli clones are implicated in a variety of diseases, including urinary tract infections (UTIs), a leading cause of human antibiotic use (24-28). The spread of multidrug-resistant E. coli is now a major health concern, especially the pandemic fimH30 subclone of sequence type 131 (ST131) (H30). Though recently emerged, $\mathrm{H} 30$ is now globally distributed and comprises up to half of all urinary and bloodstream isolates of $E$. coli that are fluoroquinolone resistant and produce extended-spectrum beta-lactamases (ESBL) (29-31).

Additionally, H30 is strongly associated with "drug-bug" mismatches and adverse outcomes in elderly and immunocompromised individuals $(29,30)$. Somewhat paradoxically, $\mathrm{H} 30$ is also a persistent gut colonizer of healthy people and frequently causes asymptomatic bacteriuria ( $A B U$ ) in such carriers (31). However, the relative clonal predominance of $\mathrm{H} 30$ strains among $E$. coli strains colonizing the gut or bladder in healthy carriers remains unknown. Answering these questions could have a significant impact on understanding the spread of antibiotic resistance and its reservoirs.

Currently, microbiome diversity is studied by sequencing the 16S rRNA gene, but this cannot capture clonal diversity $(32,33)$. Conventional methods for assessing clonal diversity, such as metagenomic sequencing and single-colony typing, are costly and labor-intensive. For reliable clonal diversity analysis, metagenomic sequencing requires very high coverage per sample, while single-colony typing requires handpicking large numbers of colonies for multilocus sequence typing (MLST) (34-37). In E. coli, MLST requires assessment of 7 genes per isolate, which is analytically complex, costly, and labor-intensive, and therefore difficult to implement. Previously, we reported an alternative clonotyping method that requires sequencing regions of only 2 genes: fumC, which is part of the MLST scheme, and fimH, which encodes a rapidly evolving fimbrial adhesin (38). The fumC-fimH-based $(\mathrm{CH})$ typing of $E$. coli is widely accepted due to its simplicity and ability to not only identify specific STs, but subdivide them into smaller subclones (38). Specifically, H3O is identified using the allele combination fumC4OfimH30, while other, less resistant ST131 strains have the same fumC but different fimH alleles.

Here, we report a high-throughput method for clonal typing of $E$. coli strains by combining $\mathrm{CH}$ typing and deep amplicon sequencing. We developed a new algorithm - population-level allele profiler (PLAP)_for detecting alleles and predicting the relative prevalence of each allele in a sample. We were able to assess the prevalences of clonal groups (including $\mathrm{H} 30$ ) in multiple fecal and urine samples concurrently, with a limit of relative abundance detection at $<1 \%$ of the total population.

\section{RESULTS}

Deep amplicon sequencing of defined samples. To validate our approach and establish a limit of detection for strain presence, we first tested our deep amplicon sequencing procedure on a set of defined samples. To create the defined samples, we first selected a fecal sample from our laboratory collection known to contain $\mathrm{H} 30$ and ST101. Next, we isolated a single colony of each and confirmed them to be strains of $\mathrm{H} 30$ (fum C40-fimH30) and ST101 (fumC41-fimH86) by using $\mathrm{CH}$ typing. From these single colonies, we first created H30-only and ST101-only mixtures of fumC and fimH amplicons. We also created four ST101-H30 mixed samples by combining the fumC and fimH amplicons from ST101 and H30 in ST101/H30 ratios of 1:1, 1:4, 1:100, and 1:1,000.

Analysis of raw sequencing data from $\mathrm{H} 30$-only and ST101-only samples showed the average coverage of erroneous bases was $0.08 \% \pm 0.09 \%$ for both strains. Erroneous bases were observed in both genes across most nucleotide positions. The highest coverages for an erroneous base were $0.66 \%$ of aligned reads in fumC and $0.45 \%$ in fimH for $\mathrm{H} 30$ and $0.68 \%$ of reads in fumC and $0.46 \%$ in fimH for ST101. The frequency 


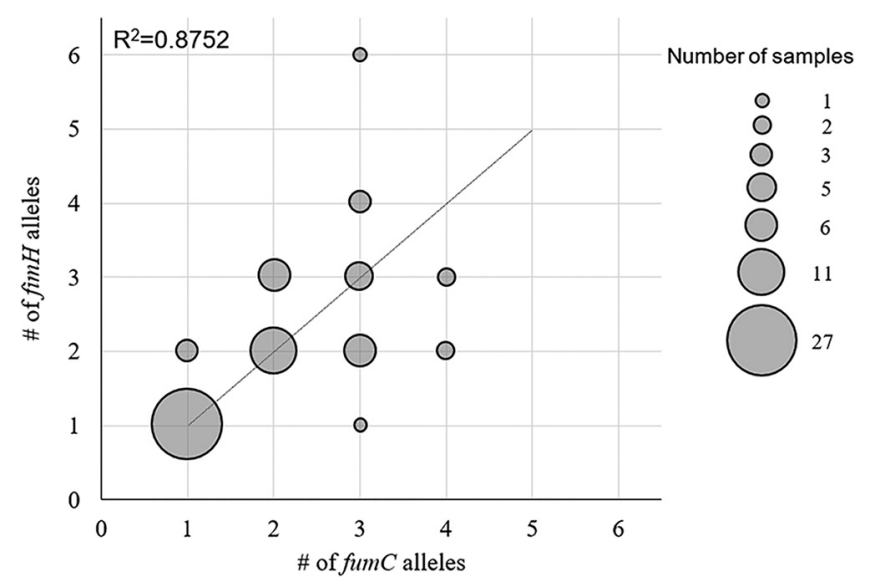

FIG 1 Congruency of fumC and fimH allele counts in fecal and urine samples. The sizes of the circles correspond to the numbers of samples with designated fumC or fimH allele counts (i.e., 1 sample with one fum C allele and three fimH alleles). The linear fit with the Pearson square correlation index is shown.

distribution for erroneous base coverage is presented in Fig. S1 in the supplemental material.

Analysis of raw sequencing data from ST101-H30 mixtures showed that both $\mathrm{H} 30$ and ST101 alleles were detectable in the 1:1, 1:4, and 1:100 mixtures. In the 1:1,000 mixture, only alleles of the dominant $\mathrm{H} 30$ strain were observed. In the 1:1, 1:4, and 1:100 mixtures, the input and observed allele prevalence were highly correlated for both fumC and fimH ( $R^{2}=0.996$ and 0.997 , respectively [see Fig. S2 in the supplemental material]). Erroneous bases were observed at $0.09 \% \pm 0.1 \%$ and $0.08 \% \pm 0.09 \%$ of aligned reads in fumC and $\mathrm{fimH}$, respectively (see Fig. S1). The highest coverages for erroneous bases among all mixtures were $0.79 \%$ of aligned reads for fumC and $0.57 \%$ of aligned reads for fimH.

Since $0.79 \%$ of aligned reads was the highest coverage for an erroneous base, we established $0.8 \%$ as a cutoff for correct base calling in both genes. This cutoff was used for all further PLAP analysis.

Deep sequencing of study samples and allele prediction. Next, we applied PLAP to 67 participant samples (43 fecal and 24 urine) collected from a previous study (31). A total of 128 fumC and $129 \mathrm{fimH}$ alleles were predicted across all the samples, of which $123(96.1 \%)$ and 125 (96.9\%) were previously known fumC and fimH alleles, respectively. Five novel fum $\mathrm{C}$ and 4 novel fimH alleles were potentially detected. All the novel fumC and $f i m H$ alleles were phylogenetically distant from other alleles predicted in the sample, indicating that the alleles are not artifacts of sequencing (see Fig. S3 and S4 in the supplemental material). These novel alleles nonetheless clustered with other $E$. coli fum $C$ and $\mathrm{fimH}$ alleles, indicating that they are novel $E$. coli alleles rather than alleles belonging to other species.

The average number of alleles predicted per sample was $1.91 \pm 0.96$ for fumC and $1.93 \pm 1.01$ for fimH. Forty-three samples had the same numbers of predicted fum $C$ and fimH alleles; 24 samples had different numbers of predicted fum $\mathrm{C}$ and $\mathrm{fimH}$ alleles (Fig. 1). Overall, the number of predicted fumC alleles correlated with the number of predicted fimH alleles, with an $R^{2}$ value of 0.88 (Fig. 1).

To assess the performance of PLAP for predicting alleles, we used samples containing criterion clones-strains previously identified by single-colony typing. PLAP detected criterion fimH and fumC alleles in 52 of the samples (90\%). In the 6 samples where a criterion allele(s) was not found, the criterion clones were ciprofloxacin resistant, but their isolation from the sample required $\geq 2$ plating attempts. This leads us to believe that these alleles were not detected because they were absent in the MacConkey agar-plated population prior to deep sequencing.

A total of 72 noncriterion (previously unidentified) fumC and 71 noncriterion fimH alleles were predicted by PLAP across all 67 samples. To assess the performance of PLAP 

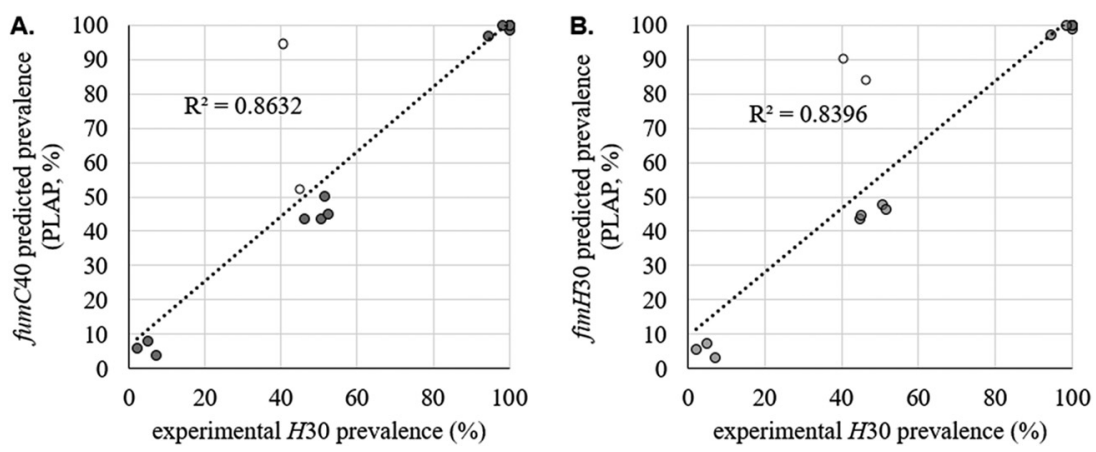

FIG 2 Validation of predicted H30 allele prevalence. Shown are the PLAP-predicted prevalences of H30 alleles versus actual $\mathrm{H} 30$ loads in $\mathrm{H} 30$-containing fecal samples. The predicted prevalences of fumC40 (A) and $\mathrm{fimH} 30$ (B) are shown. The predicted prevalences of fum $\mathrm{C} 40$ and fimH3O are expressed as percentages of all $E$. coli bacteria in each sample. The experimentally confirmed $\mathrm{H} 30$ load is expressed as the percentage of $\mathrm{H} 30$ (ciprofloxacin-resistant) single colonies among all plated $E$. coli single colonies. At least 130 colonies were tested per sample. Outliers (open circles) were outside the $99 \%$ confidence interval of the number of colonies tested.

on noncriterion alleles, we analyzed 14 samples (10 fecal and 4 urine) predicted to contain 22 noncriterion fum $\mathrm{C}$ and 22 noncriterion fimH alleles. Twelve of these samples had at least one noncriterion allele alongside criterion alleles; the remaining 2 had multiple noncriterion alleles only in each gene. For each sample, $\geq 40$ single colonies were isolated, and the $\mathrm{CH}$ type was determined using 7-single-nucleotide polymorphism (SNP) quantitative PCR (qPCR), with each $\mathrm{CH}$ type verified by sequencing. With these data, we confirmed 19 (86\%) predicted noncriterion alleles for each gene. They included one predicted novel fumC allele. Of the unconfirmed alleles, one was not distinguishable by 7-SNP qPCR and had a predicted prevalence of $1 \%$; therefore, we did not attempt to locate it. The remaining unconfirmed alleles had predicted prevalences of $<3 \%$ and therefore may have been missed due to insufficient sampling. Additionally, all the criterion alleles in these samples, 12 per gene, were predicted by PLAP.

Prediction of allele prevalence in multiallele samples. We have also designed PLAP to predict the within-sample prevalence of each allele. The average allele prevalence in fecal samples was $47.3 \% \pm 4.3 \%$ (standard error of the mean [SEM]) (range, $0.88 \%$ to $100 \%$ ) for fumC and $48.4 \% \pm 4.22 \%$ (SEM) (range, $1 \%$ to $100 \%$ ) in $\mathrm{fimH}$. The average allele prevalence in urine samples was $64.8 \% \pm 6.91 \%$ (SEM) (range, $1.4 \%$ to $100 \%$ ) for fumC and $58.3 \% \pm 7.18 \%$ (SEM) (range, $1 \%$ to $100 \%$ ) in $\mathrm{fimH}$.

In order to verify that the prevalences predicted by PLAP were accurate, we compared the predictions to actual in-sample prevalences using two different methods.

In the first method, we used $\mathrm{H} 30$, since ascertaining its prevalence is relatively simple. By plating the sample on MacConkey agar and then patching onto LBciprofloxacin, it is possible to compare the number of ciprofloxacin-resistant $(\mathrm{H} 30)$ colonies to the total number of $E$. coli colonies. The ratio of these two numbers provides the $\mathrm{H} 30$ load in a sample. We compared the predicted prevalences of fumC4O and fim $\mathrm{H} 30$ to the $\mathrm{H} 30$ load in 17 fecal samples containing ciprofloxacin-resistant $\mathrm{H} 30$.

The correlations between the $\mathrm{H} 30$ load and the predicted prevalences of fumC 40 and $\mathrm{fimH} 30$ were 0.86 and 0.84 , respectively (Fig. 2), indicating that the prevalences given by PLAP were representative of actual allele prevalences. To determine whether outliers were present, we calculated the $99 \%$ confidence interval $(\mathrm{Cl})$ range for every sample (see Materials and Methods). Three outlier samples were identified (Fig. 2, open circles). Since it is possible that these outliers contain ciprofloxacin-sensitive non- $\mathrm{H} 30$ fimH30-containing clones, fumC-null or fimH-null clones, and/or ciprofloxacin-sensitive $\mathrm{H} 30$, we decided to employ screening of a large number of single colonies.

In the second method, we used single-colony typing for the in-depth characterization of 14 multiallele samples described above, alongside 4 additional single-allele samples ( 2 fecal and 2 urine) for which only one allele per gene was predicted. This set 

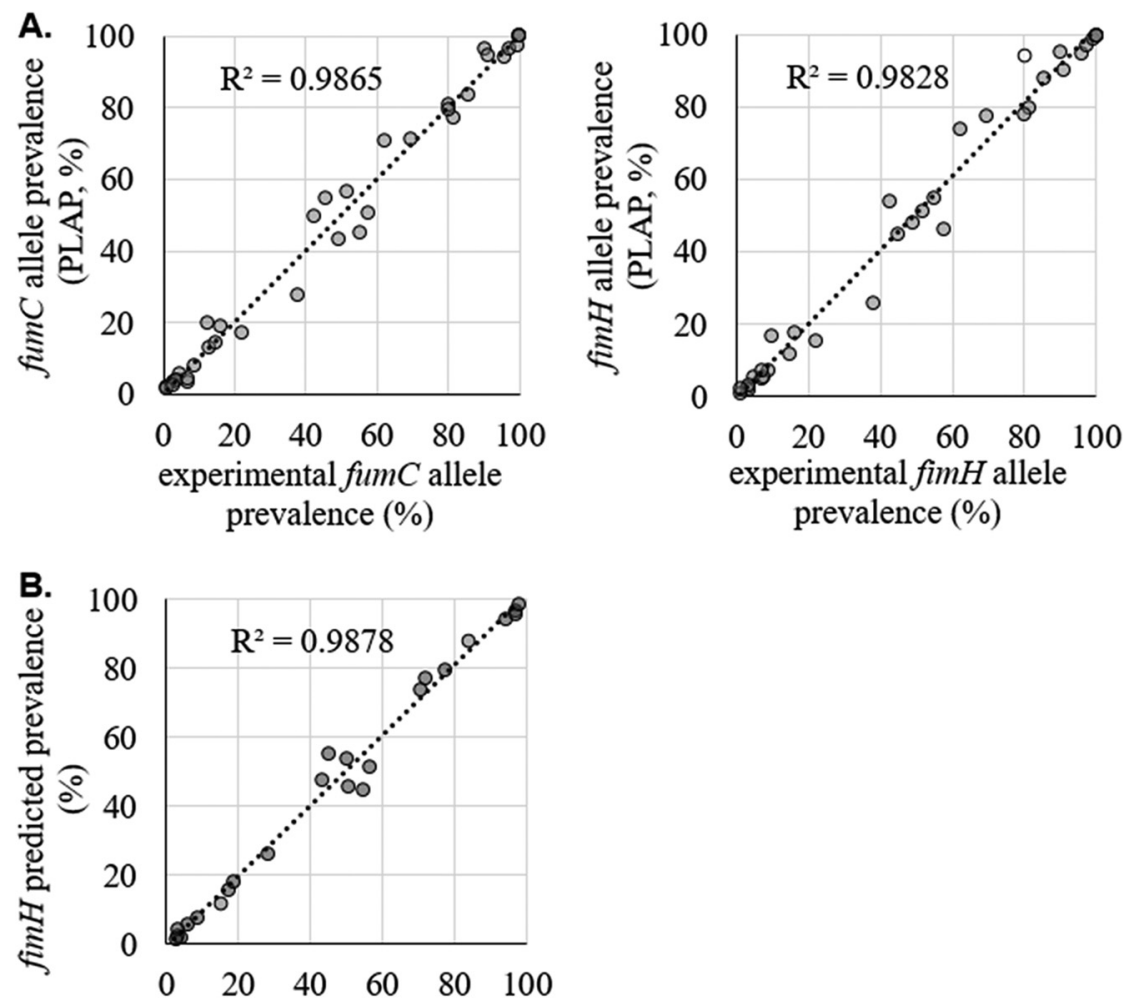

\section{fumC predicted prevalence (\%)}

FIG 3 Validation of predicted fumC and fimH allele prevalences. (A) PLAP-predicted versus experimental within-sample fum $\mathrm{C}$ and $\mathrm{fimH}$ allele prevalences in 18 samples. Experimental allele prevalence was determined by $\mathrm{CH}$ typing of at least 40 single bacterial colonies per sample. Outliers (open circles) were outside the $99 \%$ confidence interval of the number of colonies sampled. (B) Predicted prevalence of fumC versus fimH alleles from the same $\mathrm{CH}$ type in 11 samples where no sharing of alleles between strains was present.

of 18 samples included 11 of the 17 fecal samples used for the H30-based analysis described above, including one of the outlier samples. For all 18 samples, we used $\mathrm{CH}$ typing of $\geq 40$ single colonies per sample to determine the prevalence of each fumC and $\mathrm{fimH}$ allele. Correlation between the PLAP-predicted prevalence and the experimental allele prevalence was 0.98 for both fumC and fimH alleles (Fig. 3). As in the H3O analysis described above, we determined whether outliers were present by using the $99 \% \mathrm{Cl}$ range for every sample. Only one outlier was detected, corresponding to the only sample that contained colonies from which fimH could not be amplified (fimH-null colonies). Furthermore, the sample that was an outlier in the H30-based analysis was found to contain a relatively rare ciprofloxacin-sensitive $\mathrm{H} 30$.

Matching fum C and fimH alleles to predict sample strain content. In $\mathrm{CH}$ typing, unique combinations of fum C and fimH alleles are used to determine the identities of the strains in a sample. Since a strain contains one copy of fumC and fimH, the prevalences of alleles of the two genes in the sequencing data should be identical. For example, in a sample containing 30\% H30 (fumC40-fimH30) and 70\% ST101 (fumC41fimH86), we expect to see $30 \%$ of fumC reads to be fumC40 and $30 \%$ of fimH reads to be fimH30. In reality, however, the prevalences will be slightly different due to PCR and sequencing errors. To establish an acceptable difference between the prevalences of same-strain fum $\mathrm{C}$ and fimH alleles, we looked at 11 samples containing unique $\mathrm{CH}$ types (i.e., without allele sharing). In these 11 samples, the predicted prevalences of fumC and fimH were highly correlated (0.99) (Fig. 3). First, we calculated the absolute difference between the predicted fum $\mathrm{C}$ and $\mathrm{fimH}$ prevalences for each matched pair of alleles. Next, each absolute difference was divided by the predicted fumC or fimH prevalence 

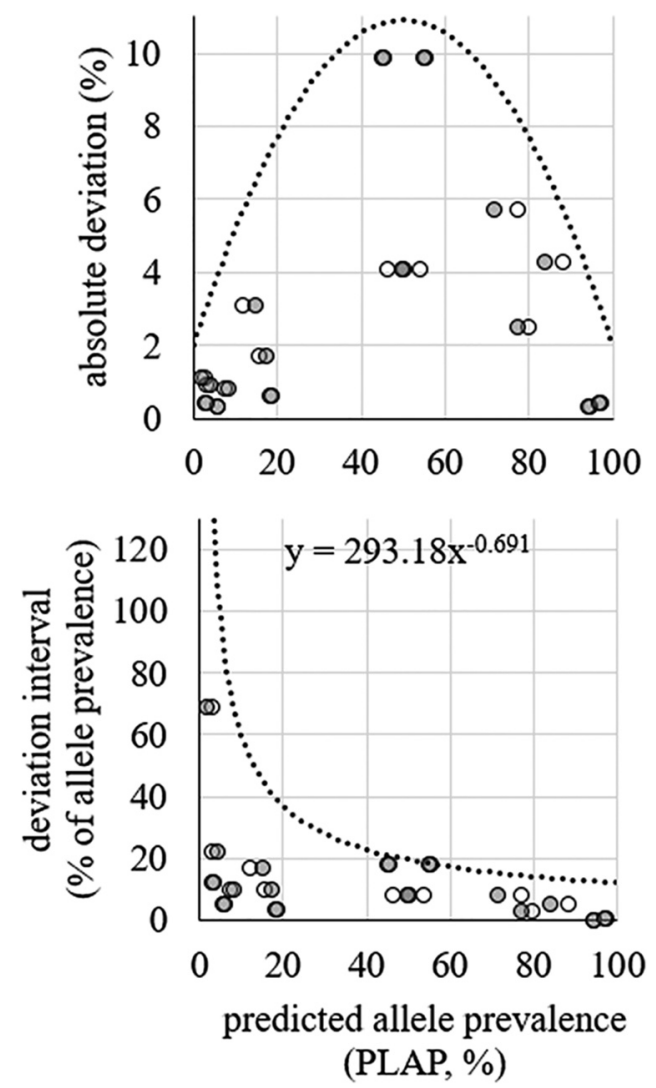

FIG 4 Difference in predicted prevalence between fumC and fimH alleles from the same $E$. coli strain. Deviation in absolute numbers (top) and deviation as a percentage of the prevalence of the allele (bottom) are shown. The open circles indicate fimH data points. The shaded circles indicate fumC data points. Trend lines and equations were used to determine intervals for matching (i.e., belonging to the same $\mathrm{CH}$ type) fum $\mathrm{C}$ and $\mathrm{fimH}$ alleles.

to obtain a relative deviation (Fig. 4). Finally, we used the relative deviations to derive an equation for the maximum acceptable difference between matching fumC and fimH alleles (Fig. 4).

While some samples, like those discussed above, contain only unique $\mathrm{CH}$ types, others contain $\mathrm{CH}$ types with shared alleles. For example, in a sample containing $30 \%$ $\mathrm{H} 30$ and $70 \% \mathrm{ST} 131$, which share fumC40, the prevalence of fumC40 is not representative of either $\mathrm{H} 30$ or ST131 prevalence. For such samples, the minority rule was applied to resolve the strain content. Thus, under the minority rule, the percentage of $\mathrm{H} 30$ in the example above would be determined by fimH3O rather than fumC40, since the fimH3O prevalence is lower. We tested this approach on both the $\mathrm{H} 30$ and the 18-sample analyses described above to see if it resolved outliers. In both cases, using the minority rule removed outliers and improved the correlation between predicted and experimental prevalence (see Fig. S5 in the supplemental material). Thus, we were able to assign strain content and strain prevalence to all samples, including samples with allele sharing.

Predicted strain diversity of fecal and urine samples. Using the equation described above, we were able to classify all the samples in our study into 4 categories (Fig. 5): samples with only one $\mathrm{CH}$ type (uniclonal); samples with multiple unique $\mathrm{CH}$ types (unambiguous); samples with one dominant unique $\mathrm{CH}$ type and multiple minor, nonunique $\mathrm{CH}$ types (ambiguous-simple); and samples where the dominant $\mathrm{CH}$ type was not unique (ambiguous-complex). Fecal samples were 33\% uniclonal, 23\% unambiguous, $21 \%$ ambiguous-simple, and 23\% ambiguous-complex. Urine samples were $54 \%$ uniclonal, $8 \%$ unambiguous, 25\% ambiguous-simple, and $12.5 \%$ ambiguouscomplex. 


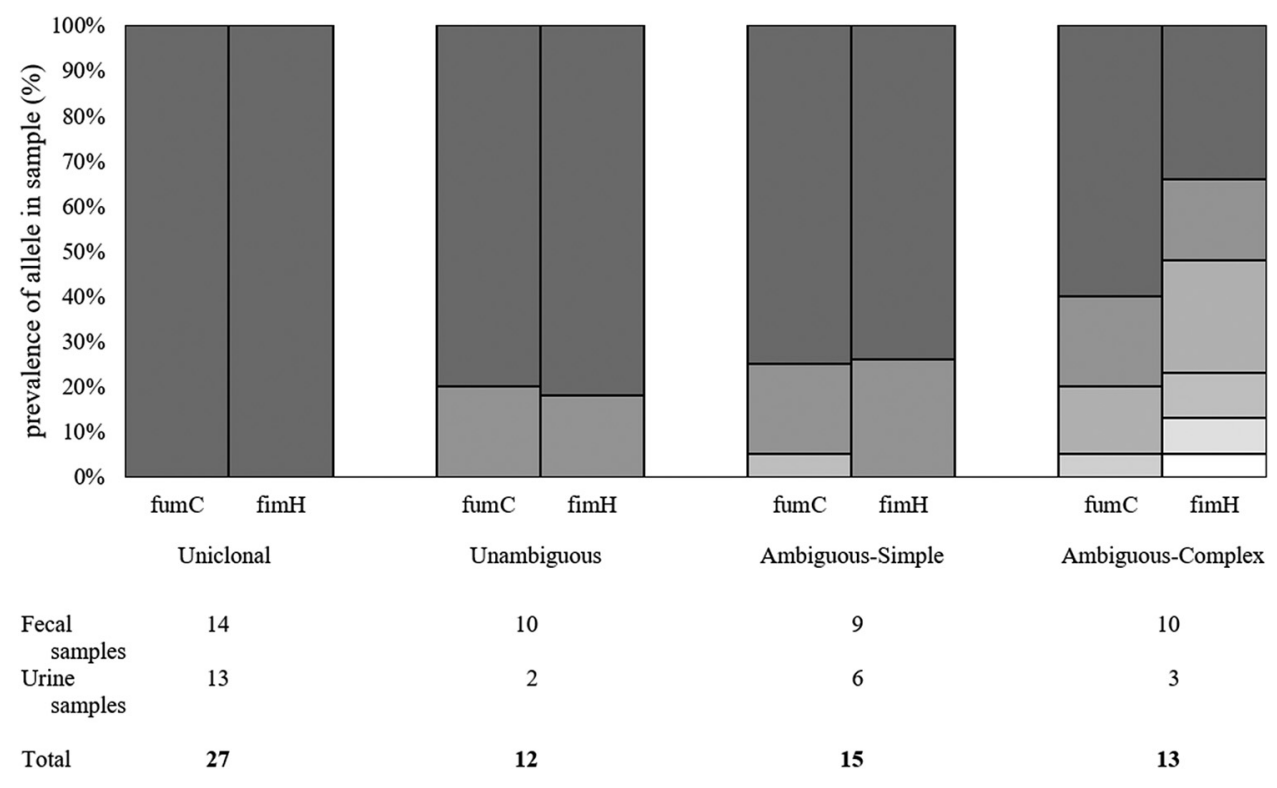

FIG 5 Representative examples of each sample category defined by within-sample breakdown of prevalence for fum $\mathrm{C}$ and fimH alleles. The numbers of fecal and urine samples belonging to each category are listed below.

Overall, $107 \mathrm{fecal}$ and 48 urine strains were predicted, corresponding to 68 clones in fecal samples and 33 clones in urine samples. Of these clones, 50 (73.5\%) and 24 (73\%), respectively, were found in Enterobase, an online repository of $E$. coli genomes and MLST types (https://enterobase.warwick.ac.uk).

Out of the 155 total strains predicted, 6 were fumC null (3.9\%) and 2 were fimH null (1.3\%). This is congruent with the occurrence of null alleles in our 18-sample subset, where $1(3 \%)$ out of 35 total strains predicted was a null-allele strain.

The average number of strains per sample was $2.47 \pm 1.32$ for fecal samples and $1.96 \pm 1.40$ for urine samples. Based on Enterobase's ST-phylogroup data, we determined that B2 was the most common (14 out of $47 ; 30 \%$ ) among noncriterion fecal strains. Other phylogroups included A (26\%), B1 (19\%), C (8.5\%), D (11\%), E (2\%), and $F(4 \%)$. Noncriterion strains in urine samples included strains from phylogroups B2 (8 out of $16 ; 50 \%), B 1(19 \%), D(19 \%)$, and $A$ and $F(6 \%$ each).

Novel clones. Seventeen fecal samples (40\%) and 8 urine samples (33\%) in our study were found to contain at least one novel $\mathrm{CH}$ type. They included 19 fecal and 9 urine $\mathrm{CH}$ types not found in Enterobase. Of these, $5 \mathrm{fecal}$ and 3 urine $\mathrm{CH}$ types included at least one novel allele, and 14 fecal and 6 urine $\mathrm{CH}$ types were combinations of fumC and $\mathrm{fimH}$ that were not previously observed (novel $\mathrm{CH}$ combinations). Both $\mathrm{CH}$ types involving novel alleles and novel $\mathrm{CH}$ combinations were observed to be primarily low-frequency clones. The average predicted prevalence for novel $\mathrm{CH}$ combinations was $8.7 \% \pm 3.5 \%$ (SEM) (range, $1 \%$ to $64.2 \%$ ), and 13 out of 20 novel $\mathrm{CH}$ combinations had predicted prevalences of $<5 \%$. One such combination was confirmed in our set of 14 characterized samples, consisting of fumC24 and fimH9, with a predicted prevalence of $1.6 \%$ and experimental prevalence of $1.2 \%$.

Similarly, 7 out of 8 novel allele-containing $\mathrm{CH}$ types had predicted prevalences of $<2 \%$. The remaining $\mathrm{CH}$ type had a predicted prevalence of $70.7 \%$ and was detected using single-colony typing. The novel fumC allele was paired with fimH47 and was verified to be 8 SNPs away from the closest known allele. The remaining MLST gene alleles for the strain were adk46, icd260, mdh160, gyrB266, purA1, and recA221.

Clones below the error threshold. To ascertain if we could identify alleles at prevalences below our defined error threshold of $0.8 \%$, we ran PLAP on the set of 14 multiallele samples, using an error threshold of $0.5 \%$. In 8 and 6 samples, respectively, the prevalence of fumC and fimH alleles was $<0.8 \%$. None of the alleles corresponded 


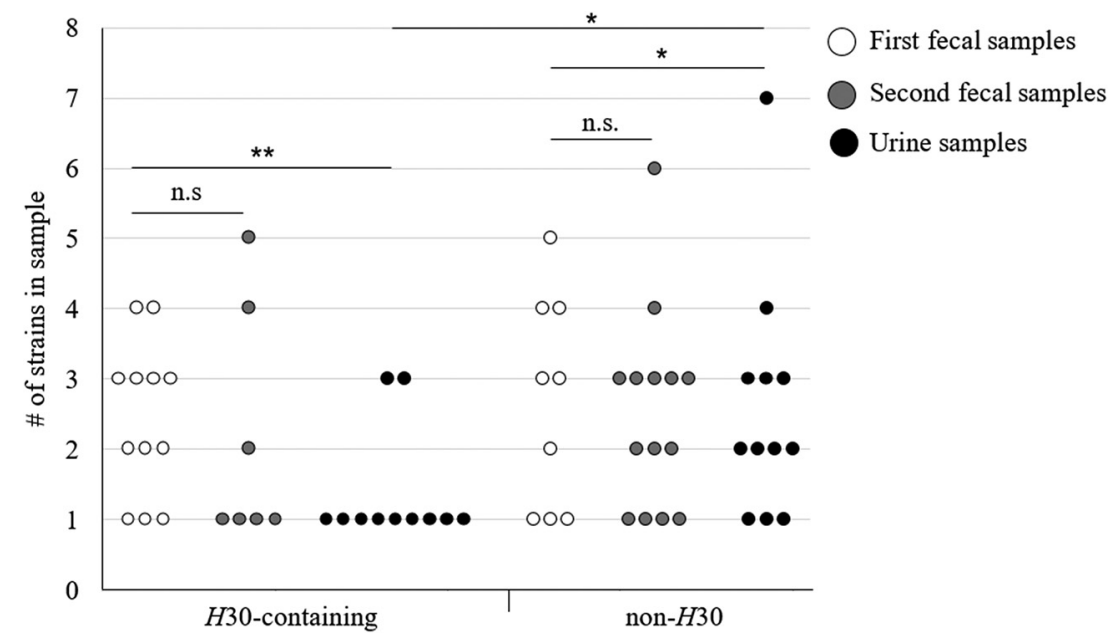

FIG 6 Diversity of $E$. coli in individual fecal/urine samples. H30 content was determined by PLAP and/or culturing.

to known fumC or fimH alleles. These apparently novel alleles clustered alongside known alleles identified in the sample (see Fig. S6 and S7 in the supplemental material), leading us to conclude that they arose due to sequencing or amplification errors rather than belonging to clonally different strains.

Predicted strain diversity in urine and fecal samples. Strain diversity in the first fecal samples was comparable to the diversity in the second fecal samples (paired $t$ test; $P>0.1$ ). Distinguishing between $\mathrm{H} 30$-containing and non- $\mathrm{H} 30$ samples showed that there was no statistical difference in strain diversity between $\mathrm{H} 30$-containing and non-H30 fecal samples of either kind (unpaired $t$ test; $P>0.1$ ) and that there was no difference in diversity between first and second fecal samples in either non- $\mathrm{H} 30$ or H30-containing samples (Fig. 6) (paired $t$ test; $P>0.1$ ). Both $\mathrm{H} 30$ and non-H30 urine samples were less diverse than corresponding fecal samples (paired $t$ test; $P<0.01$ and 0.02 , respectively). However, $\mathrm{H} 30$ urine samples were less diverse than non- $\mathrm{H} 30$ urine samples ( $t$ test; $P=0.04$ ).

It is also noteworthy that in 6 out of $23 \mathrm{H} 30$-containing fecal samples, $\mathrm{H} 30$ was the only strain predicted, indicating that it may be fully dominant in the gut niche in these participants.

Strain turnover in fecal samples. There was no correlation between the numbers of strains in the first and second fecal samples, as well as no correlation between the numbers of strains in the urine sample and either fecal sample (Fig. 7). When comparing the strain contents of the first and second fecal samples, we found that $92 \%$ of noncriterion strains appeared to be transient, i.e., were detected in one of the fecal samples only. Transient noncriterion strains were also skewed toward lower-frequency strains ( $t$ test; $P<0.001$ ) (Fig. $8 \mathrm{~B}$ ). It is possible that these strains were present in both fecal samples but were below our limit of detection in one. However, we found that in one participant (P2 [see the supplemental material]) the first fecal sample contained 3 ciprofloxacin-sensitive noncriterion strains while the second fecal sample contained only ciprofloxacin-resistant $\mathrm{H} 30$, as verified by single-colony testing. This leads us to believe that there may have been significant strain turnover in our fecal samples overall.

\section{DISCUSSION}

We combined conventional fumC-fimH typing with deep amplicon sequencing to assess $E$. coli clonal diversity in a high-throughput manner. Our method has several advantages over existing protocols. First, our method has high sequencing resolution for target species. Since we sequence only $E$. coli fumC and fimH, we can generate $\geq 0.5$ million reads per sample, yielding $\geq 5,000$ reads per base. In contrast, metagenomic 

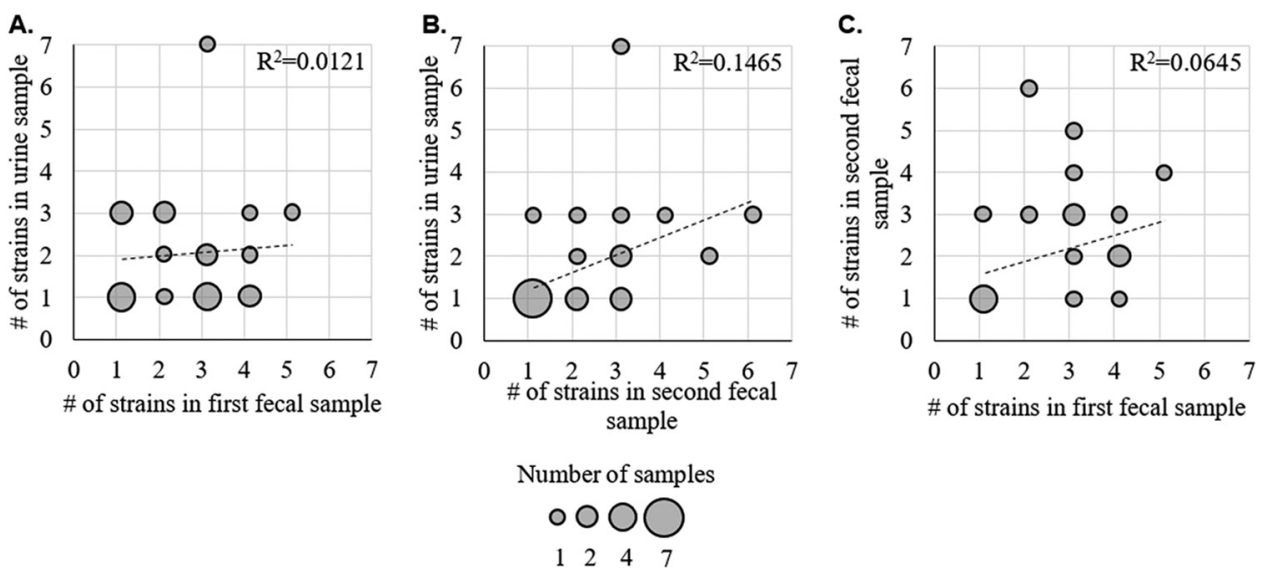

FIG 7 Counts of $E$. coli strains in fecal and urine samples. Shown are the numbers of strains detected by PLAP in the first fecal sample versus urine (A), second fecal sample versus urine (B), and first fecal sample versus second fecal sample (C). Each circle represents participants with the corresponding number of $E$. coli strains in the designated sample. The circle size indicates the number of participants with the determined number of strains. The linear fit with the Pearson square correlation index shown.

sequencing, which is nonspecific for target species, yields only 20 reads per base per genome (assuming a 5-Mb genome). Secondly, our method assessed up to 46 samples per sequencing run. In contrast, MLST requires typing $\geq 100$ single colonies per sample to capture the low-prevalence strains that PLAP detects. Finally, while we developed PLAP for E. coli $\mathrm{CH}$ typing, PLAP is not limited to $E$. coli clonotyping and may be generalized to other MLST schemes. For those attempting to use or adapt our approach, we have provided guidelines for both the experimental and algorithm portions on PLAP's website (http://www.github.com/marade/PLAP).

Despite studies showing that the healthy gut E. coli population typically includes multiple clones, we show that the pandemic multidrug-resistant subclone $\mathrm{H} 30$ can dominate the gut in healthy women, sometimes as the only detectable clone $(42$, 44-47). This builds upon previous research that has found multidrug-resistant bacteria in healthy people and healthy people who appear to harbor only one gut clone $(42,46)$. Total dominance is especially concerning, since antibiotic pressure was absent, indicating that $\mathrm{H} 30$ is potentially outcompeting other clones by alternative means. Whether these mech-
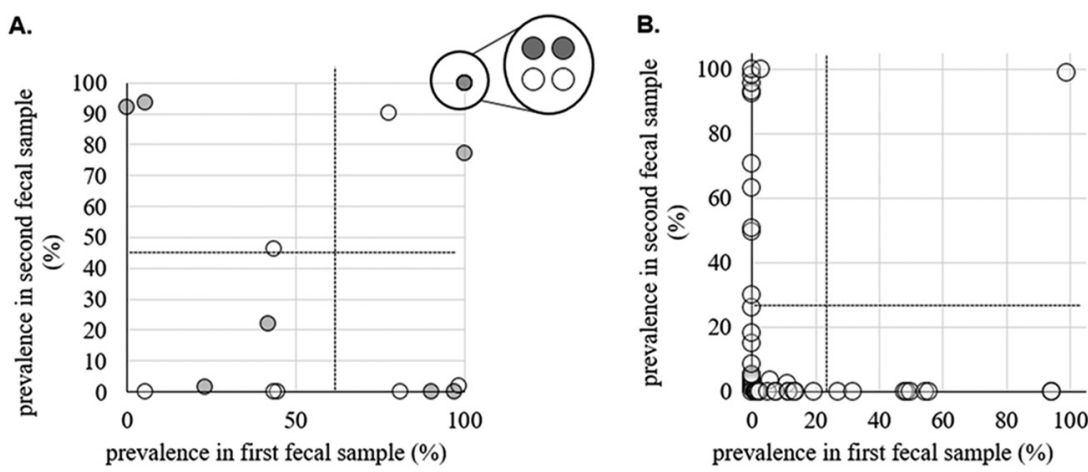

FIG 8 Persistence of $E$. coli strains in fecal samples. (A) Prevalences of criterion fecal strains in first versus second fecal samples. The open data points represent $\mathrm{H} 30$ strains, while the shaded data points represent non-H30 strains. The circled cluster represents 4 strains present at $100 \%$ prevalence in both samples. The dotted lines indicate the mean prevalence for strains in the first and second fecal samples. The distribution of prevalences in both first and second fecal samples is not significantly different from random ( $t$ test; $P>0.05$ ). (B) Prevalences of noncriterion fecal strains in first versus second fecal samples. The dotted lines indicate the mean prevalence for transient strains in the first and second fecal samples. Transient strains are defined as strains that are present in only one of the two fecal samples from the same participant. The distribution of prevalences in both first and second fecal samples is significantly skewed toward lower prevalences ( $t$ test; $P<0.01$ ). 
anisms are metabolic or whether certain virulence factors give $\mathrm{H} 30$ an advantage is unclear, though previous studies have speculated that some virulence factors may be beneficial for E. coli gut survival $(40,43,44)$. Additionally, our study involved a small number of participants in whom $\mathrm{H} 30$ was present in the gut and bladder. Therefore, it is possible that host differences play a significant role. Another novel observation was that $\mathrm{H} 30$ was the sole detected urinary strain more frequently than other clones, regardless of $\mathrm{H} 30$ gut dominance or nondominance. This may indicate that $\mathrm{H} 30$ is an especially well-adapted uropathogen, potentially explaining its association with UTI. Since it is unknown how ABU converts to UTI, further study of H30 dominance in both $A B U$ and UTI is needed.

We also uncovered substantial diversity in our samples. This included significant $E$. coli diversity in non-H30 urine samples from healthy women. Reports of multistrain bacteriuria are rare, likely due to the convention of selecting one isolate per urine sample $(41,42)$. Therefore, it is unknown how common multistrain bacteriuria may truly be. Remarkably, we also detected low-prevalence strains in the gut, some of which were novel clones, with up to 6 clones in a single sample. Gut $E$. coli diversity of this magnitude is supported by studies typing $>200$ single colonies per sample (42). Studies using smaller counts usually report fewer clones, indicating that there may be undescribed E. coli diversity when manageable numbers of colonies are used $(40,41,47)$. Therefore, we believe that microbiome-like approaches to $E$. coli diversity are necessary to fully understand intraspecies dynamics in both the gut and bladder.

Our approach does have limitations. First, our lowest detectable strain prevalence is $0.8 \%$ of the $E$. coli population. This limit may be addressed in several ways, including use of a high-fidelity polymerase and preferential selection of $E$. coli colonies. However, we also recognize that detection of rare strains may still prove difficult and that methods like ours may not fully replace current techniques. Secondly, our method relies on subculturing E. coli. We are aware that, theoretically, some strains could be suppressed during growth on selective media, forming no or smaller colonies and skewing prevalence results. However, we did not encounter this during our study. While amplification of fumC and fimH may be applied to urine samples without culturing, attempts at doing this directly from fecal samples were unsuccessful, possibly due to $E$. coli comprising $<1 \%$ of the gut microbiome, making $E$. coli DNA too rare to effectively amplify. Therefore, we used culturing for all samples and believe that evaluating target species abundance using $16 \mathrm{~S}$ rRNA sequencing is warranted in such cases. Lastly, we used antibiotic resistance for validation, which is not possible with clones/species where antibiotic resistance is absent or not strongly clonal. In these cases, validation using single-colony typing should be considered. These issues lower the reliability of our approach, but we believe that it remains an important step toward development of comprehensive clonal diversity (clonobiome) assessment tools for any species of interest.

\section{MATERIALS AND METHODS}

Study design and sample processing. We selected a subset of participants from a previous study carried out by Kaiser Permanente Washington and the University of Washington (Seattle, WA) (31). That study identified healthy gut carriers of ciprofloxacin-resistant E. coli, including E. coli $\mathrm{H} 30$. These E. coli strains were found in initial fecal samples by plating on LB-ciprofloxacin and CH typing of 1 to 8 single colonies. After the initial fecal sample was analyzed, the $\mathrm{H} 30$ carriers, as well as carriers of some other strains, were asked to provide urine samples. These were received on average $152 \pm 55.9$ days after the initial sample ( $85 \%$ responded). The respondents were then asked to provide follow-up fecal samples, which were received on average $82 \pm 41.1$ days after the urine sample ( $84 \%$ responded). All the fecal and urine samples were tested for ciprofloxacin-resistant $E$. coli as with the initial samples. For this study, we chose 28 individuals who supplied all three samples. For 11 participants, H30 was identified in all three samples; for 4 additional participants, H30 was isolated in two samples. For 8 participants, ciprofloxacinresistant ST1193 was found in at least two samples. For 5 participants, the same ciprofloxacin-susceptible clone was found in at least two samples. The sample types, strain clonal identities, and sampling times for all participants are shown in Fig. S8 in the supplemental material. The average age of participants was $66.7 \pm 15.7$ years.

Preparation of predefined control samples. For control experiments, two predefined strains were chosen: $\mathrm{H} 30$ (E. coli FESS614.ds6) and clonal group ST101 (E. coli FESS614.ds4). DNA from these strains was extracted, and fumC and fimH were amplified by PCR under the following conditions: 3 min 
denaturation $\left(95^{\circ} \mathrm{C}\right), 35$ cycles of annealing $\left(95^{\circ} \mathrm{C}\right.$ for $45 \mathrm{~s}, 57^{\circ} \mathrm{C}$ for $45 \mathrm{~s}$, and $72^{\circ} \mathrm{C}$ for $\left.45 \mathrm{~s}\right), 5$ min extension $\left(72^{\circ} \mathrm{C}\right)$, and $4^{\circ} \mathrm{C}$ hold. The primers $(10 \mu \mathrm{M})$ used were as follows: $5^{\prime}$-TCACAGGTCGCCAGCGCTTC-3' (fumC forward), 5'-GTACGCAGCGAAAAAGATTC-3' (fumC reverse), 5'-TCAGGGAACCATTCAGGCA-3' (fimH forward), and 5'-ACAAAGGGCTAACGTGCAG-3' (fimH reverse). The amount of PCR product was measured with Qbit. To create H30-only and ST101-only samples, the corresponding fumC and fimH PCR products were pooled at a 1:1 ratio. To create mixtures, H30 and ST101 amplicons of fumC were mixed in ST101/H30 ratios of 1:1, 1:4, 1:10, 1:100, and 1:1,000. The same was performed with fimH amplicons. The fum $\mathrm{C}$ and $\mathrm{fimH}$ mixtures were then pooled by ratio type to create mixtures that had equal concentrations of total fumC and fimH. The DNA mixtures were prepared for sequencing with a Nextera XT DNA library preparation kit using the standard protocol. The resulting library was sequenced on the Illumina MiSeq (v3 kit). All the mixtures, except 1:10, reached coverage of $\geq 9,000 \times$ and were analyzed.

Deep sequencing and allele analysis of fecal and urine samples. Each fecal and urine sample was plated on MacConkey agar to reach $\sim 1,000$ E. coli single colonies per plate. All the colonies were swabbed from the agar, and DNA was extracted using a Qiagen blood and tissue kit. From this pooled DNA, fumC and fimH genes were amplified by PCR using the same primers and conditions described above for control samples. The amplicons were then purified and pooled by sample using a Qiagen gel extraction kit and then prepared for sequencing with a Nextera XT DNA library preparation kit using the standard protocol except for the use of $52.5 \mu \mathrm{l}$ of resuspension buffer (RSB) in the final magnetic-bead cleanup step. The resulting library was sequenced on the Illumina MiSeq (v3 kit). Sequencing data were analyzed using a Python program of our construction, PLAP, and have been made available for public use on GitHub (http://www.github.com/marade/PLAP). The process is described below (see Fig. $\mathrm{S9}$ in the supplemental material).

For each sample, adapter sequences were removed using Trim-Galore, and the resulting trimmed reads were aligned to a list of all known fumC and fimH alleles using KMA with strict $99.99 \%$ identity matching (https://github.com/FelixKrueger/TrimGalore) $(48,49,50)$. For each KMA-detected allele per sample, trimmed reads were again aligned to the sequence using Minimap2 and SAMtools $(49,50)$. Any candidate allele that had at least 1 base supported by $<0.8 \%$ of reads was removed from consideration. False positives were filtered using a moving 10-bp window for each allele as follows. Reads of $\geq 100 \mathrm{bp}$ with $100 \%$ identity within the window were counted. Alleles with low initial coverage, unstable coverage (high average deviation from the mean), and high similarity in the coverage pattern to an allele with more stable coverage were removed from consideration. If $>3$ alleles were left for consideration for a gene, 10-bp moving-window analysis was repeated with $\geq 200$-bp reads. If for any interval in this second analysis $>60 \%$ of coverage was lost compared to the first moving-window coverage, the allele was discarded. Heterogeneity at any positions that remained undescribed by surviving alleles was recorded. The relative abundances of all alleles were determined using the minimum coverage found during the first moving-window analysis. In samples found by PLAP to be $\geq 50 \%$ made up of $<100$-bp reads (overtagmented samples), allele prevalence was calculated manually by ascertaining the base(s) unique to each allele and using the coverage of the base(s) to calculate prevalence.

Out of the 28 total sets of fecal and urine samples chosen for this study, at least one sample failed PCR amplification or sequencing library preparation in 4 sets, and therefore, all the samples from these sets were dropped. From the remaining 24 sets, we were able to sequence fum $\mathrm{C}$ and $\mathrm{fimH}$ in all three samples. Of those, 67 (89\%) samples—22 first fecal, 24 urine, and 21 second fecal-reached $\geq 9,000 \times$ coverage per gene and were included in the analysis.

Determining within-sample clonal group breakdown. The identities of strains present in a sample were determined by combining fum $\mathrm{C}$ and fimH allele numbers and determining the ST using Enterobase. In uniclonal and unambiguous samples, every allele had one match supported by the equation for maximum acceptable difference between same-strain fum $\mathrm{C}$ and $\mathrm{fimH}$. Therefore, these alleles formed a $\mathrm{CH}$ type based on which ST type was determined.

For ambiguous-simple samples, the most prevalent fum $\mathrm{C}$ and fimH alleles formed an equationsupported $\mathrm{CH}$ type. Any alleles that also had a single-equation-supported match were assigned to form a $\mathrm{CH}$ type. For all other alleles, Enterobase was consulted to determine which allele combinations had been observed. If the $\mathrm{CH}$ type(s) produced was between alleles that had different prevalences according to the equation, the "remaining" prevalence was calculated for the allele with the greater prevalence. This allele was then paired with an allele(s) for which an Enterobase-logged $\mathrm{CH}$ type was not available and/or any novel alleles until the remaining prevalence was consumed. If any allele(s) remained after this step, it was paired with the major allele of the opposite gene.

For ambiguous-complex samples, the most prevalent fumC and most prevalent fimH allele were assigned to the same $\mathrm{CH}$ type. The remaining prevalence was calculated for the allele with the greater prevalence and treated as an unmatched allele. From this step, we proceeded as with ambiguous-simple samples.

Determining prevalence of clonal groups by culturing. The prevalence of ciprofloxacin-resistant clones in each sample was determined by diluting $\sim 1 \mu \mathrm{l}$ of sample with $\geq 300 \mu \mathrm{l}$ of $\mathrm{H}_{2} \mathrm{O}$, plating $40 \mu \mathrm{l}$ of this dilution on MacConkey agar, picking $>130$ single E. coli colonies, patching on HardyCHROM UTI chromogenic agar (Hardy Diagnostics) to verify E. coli identity, and then patching colonies on LBciprofloxacin. The prevalences of other clonal groups were validated by plating on MacConkey agar and subsequent patching of single colonies onto HardyCHROM UTI agar to distinguish E. coli. The fumC and fimH alleles of these colonies were then determined by 7-SNP clonotyping and Sanger sequencing (51).

Statistical and phylogenetic analysis. To determine the $99 \% \mathrm{Cl}$ for the prevalence of ciprofloxacinresistant strains, the number of resistant colonies was treated as the number of successes, and the total number of picked colonies was treated as the total. To determine the $99 \% \mathrm{Cl}$ for the prevalence of 
ciprofloxacin-sensitive strains, the number of colonies of that strain was treated as the number of successes, and the total number of picked colonies was treated as the total. Confidence intervals were calculated using Stata (52). All $t$ tests were run using GraphPad.

Phylogenetic trees were constructed using MEGA7 (53). An erroneous base coverage graph was generated using seaborn (54). E. coli fumC alleles were downloaded from Enterobase MLST allele data. The E. coli fimH alleles used are publicly available (55). Escherichia fergusonii and Escherichia albertii fumC alleles were downloaded from NCBI. Klebsiella pneumoniae and Enterobacter aerogenes alleles of fimH were downloaded from the PATRIC database (http://www.patricbrc.org).

\section{SUPPLEMENTAL MATERIAL}

Supplemental material for this article may be found at https://doi.org/10.1128/AEM .01866-19.

SUPPLEMENTAL FILE 1, PDF file, $0.4 \mathrm{MB}$.

SUPPLEMENTAL FILE 2, XLSX file, $0.02 \mathrm{MB}$.

\section{ACKNOWLEDGMENTS}

We thank the personnel of KPWARI for assistance in collection of samples and Sifang

Chen for proofreading the manuscript.

This work was supported by the National Institutes of Health (grant numbers R01Al106007 and R42 Al116114-02 to E.V.S.).

E.V.S. conceived the project and designed the experiments. D.K. performed control sample sequencing and analysis. All other sequencing, validation, and analysis were performed by S.G.S. V.T. provided study data and samples. M.R. programmed the algorithm; M.R. and S.G.S. tested and calibrated it. S.G.S. and E.V.S. wrote the manuscript with input from all of us.

\section{REFERENCES}

1. Heintz-Buschart A, Wilmes P. 2018. Human gut microbiome: function matters. Trends Microbiol 26:563-574. https://doi.org/10.1016/j.tim.2017 .11.002.

2. Caputi V, Giron MC. 2018. Microbiome-gut-brain axis and Toll-like receptors in Parkinson's disease. Int J Mol Sci 19:1689. https://doi.org/10 .3390/ijms19061689.

3. Perez-Pardo P, Hartog M, Garssen J, Kraneveld AD. 2017. Microbes tickling your tummy: the importance of the gut-brain axis in Parkinson's disease. Curr Behav Neurosci Rep 4:361-368. https://doi.org/10.1007/ s40473-017-0129-2.

4. Sanmiguel C, Gupta A, Mayer EA. 2015. Gut microbiome and obesity: a plausible explanation for obesity. Curr Obes Rep 4:250-261. https://doi .org/10.1007/s13679-015-0152-0.

5. De la Cuesta-Zuluaga J, Corrales-Agudelo V, Velásquez-Mejía EP, Carmona JA, Abad JM, Escobar JS. 2018. Gut microbiota is associated with obesity and cardiometabolic disease in a population in the midst of westernization. Sci Rep 8:11356. https://doi.org/10.1038/s41598-018 -29687-x.

6. Roszyk E, Puszczewicz M. 2017. Role of human microbiome and selected bacterial infections in the pathogenesis of rheumatoid arthritis. Reumatologia 55:242-250. https://doi.org/10.5114/reum.2017.71641.

7. Bu J, Wang Z. 2018. Cross-talk between gut microbiota and heart via the routes of metabolite and immunity. Gastroenterol Res Pract 2018: 6458094. https://doi.org/10.1155/2018/6458094.

8. Dzidic M, Boix-Amorós A, Selma-Royo M, Mira A, Collado MC. 2018. Gut microbiota and mucosal immunity in the neonate. Med Sci Basel 6:E56. https://doi.org/10.3390/medsci6030056.

9. Nunez G. 2017. Linking pathogen virulence, host immunity and the microbiota at the intestinal barrier. Keio J Med 66:14. https://doi.org/10 .2302/kjm.66-001-ABST.

10. Tenaillon O, Skurnik D, Picard B, Denamur E. 2010. The population genetics of commensal Escherichia coli. Nat Rev Microbiol 8:207-217. https://doi.org/10.1038/nrmicro2298.

11. Gordon DM, O'Brien CL, Pavli P. 2015. Escherichia coli diversity in the lower intestinal tract of humans. Environ Microbiol Rep 7:642-648. https://doi.org/10.1111/1758-2229.12300.

12. Costea PI, Coelho LP, Sunagawa S, Munch R, Huerta-Cepas J, Forslund K, Hildebrand F, Kushugulova A, Zeller G, Bork P. 2017. Subspecies in the global human gut microbiome. Mol Syst Biol 13:960. https://doi.org/10 $.15252 / \mathrm{msb} .20177589$.
13. Metwaly A, Haller D. 2019. Strain-level diversity in the gut: the $P$ copri case. Cell Host Microbe 25:349-350. https://doi.org/10.1016/j.chom .2019.02.006.

14. Zhang C, Zhao L. 2016. Strain-level dissection of the contribution of the gut microbiome to human metabolic disease. Genome Med 8:41. https://doi.org/10.1186/s13073-016-0304-1.

15. Leatham MP, Banerjee S, Autieri SM, Mercado-Lubo R, Conway T, Cohen PS. 2009. Precolonized human commensal Escherichia coli clones serve as a barrier to $E$. coli 0157:H7 growth in the streptomycin-treated mouse intestine. Infect Immun 77:2876-2886. https://doi.org/10.1128/IAI.00059-09.

16. Hecht AL, Casterline BW, Earley ZM, Goo YA, Goodlett DR, Bubeck Wardenburg J. 2016. Clone competition restricts colonization of an enteric pathogen and prevents colitis. EMBO Rep 17:1281-1291. https:// doi.org/10.15252/embr.201642282.

17. Lam LH, Monack DM. 2014. Intraspecies competition for niches in the distal gut dictate transmission during persistent Salmonella infection. PLoS Pathog 10:e1004527. https://doi.org/10.1371/journal.ppat .1004527 .

18. Sassone-Corsi M, Nuccio SP, Liu H, Hernandez D, Vu CT, Takahashi AA, Edwards RA, Raffatellu M. 2016. Microcins mediate competition among Enterobacteriaceae in the inflamed gut. Nature 540:280-283. https://doi .org/10.1038/nature20557.

19. Moreno E, Johnson JR, Perez T, Prats G, Kuskowski MA, Andreu A. 2009. Structure and urovirulence characteristics of the fecal Escherichia coli population among healthy women. Microbes Infect 11:274-280. https:// doi.org/10.1016/j.micinf.2008.12.002.

20. Bailey JK, Pinyon JL, Anantham S, Hall RM. 2010. Commensal Escherichia coli of healthy humans: a reservoir for antibiotic-resistance determinants. J Med Microb 59:1331-1339. https://doi.org/10.1099/jmm.0.022475-0.

21. Gorrie $C L$, Mirceta M, Wick RR, Judd LM, Wyres KL, Thomson NR, Strugnell RA, Pratt NF, Garlick JS, Watson KM, Hunter PC, McGloughlin SA, Spelman DW, Jenney AWJ, Holt KE. 2018. Antimicrobial-resistant Klebsiella pneumoniae carriage and infection in specialized geriatric care wards linked to acquisition in the referring hospital. Clin Infect Dis 67:161-170. https://doi.org/10.1093/cid/ciy027.

22. Li H, Zhu J. 2017. Targeted metabolic profiling rapidly differentiates Escherichia coli and Staphylococcus aureus at species and strain level. Rapid Commun Mass Spectrom 31:1669-1676. https://doi.org/10.1002/ rcm.7949. 
23. Galardini M, Koumoutsi A, Herrera-Dominguez L, Cordero Varela JA, Telzerow A, Wagih O, Wartel M, Clermont O, Denamur E, Typas A, Beltrao P. 2017. Phenotype inference in an Escherichia coli strain panel. Elife 6:e31035. https://doi.org/10.7554/eLife.31035.

24. Bevan ER, McNally A, Thomas CM, Piddock LJV, Hawkey PM. 2018. Acquisition and loss of CTX-M-producing and non-producing Escherichia coli in the fecal microbiome of travelers to South Asia. mBio 9:e02408-18. https://doi.org/10.1128/mBio.02408-18.

25. Robin F, Beyrouthy R, Bonacorsi S, Aissa N, Bret L, Brieu N, Cattoir V, Chapuis A, Chardon H, Degand N, Doucet-Populaire F, Dubois V, Fortineau N, Grillon A, Lanotte P, Leyssene D, Patry I, Podglajen I, Recule C, Ros A, Colomb-Cotinat M, Ponties V, Ploy MC, Bonnet R. 2017. Inventory of extended-spectrum- $\beta$-lactamase-producing Enterobacteriaceae in France as assessed by a multicenter study. Antimicrob Agents Chemother 61:e01911-16. https://doi.org/10.1128/AAC.01911-16.

26. Gupta M, Didwal G, Bansal S, Kaushal K, Batra N, Gautam V, Ray P. 2019. Antibiotic-resistant Enterobacteriaceae in healthy gut flora: a report from north Indian semi-urban community. Indian J Med Res 149: 276-280. https://doi.org/10.4103/ijmr.IJMR_207_18.

27. Johnson JR, Johnston B, Clabots C, Kuskowski MA, Castanheira M. 2010. Escherichia coli sequence type ST131 as the major cause of serious multidrug-resistant E. coli infections in the United States. Clin Infect Dis 51:286-294. https://doi.org/10.1086/653932.

28. Johnson JR, Tchesnokova V, Johnston B, Clabots C, Roberts PL, Billig M, Riddell K, Rogers P, Qin X, Butler-Wu S, Price LB, Aziz M, NicolasChanoine MH, Debroy C, Robicsek A, Hansen G, Urban C, Platell J, Trott DJ, Zhanel G, Weissman SJ, Cookson BT, Fang FC, Limaye AP, Scholes D, Chattopadhyay S, Hooper DC, Sokurenko EV. 2013. Abrupt emergence of a single dominant multidrug-resistant clone of Escherichia coli. J Infect Dis 207:919-928. https://doi.org/10.1093/infdis/jis933.

29. Burgess MJ, Johnson JR, Porter SB, Johnston B, Clabots C, Lahr BD, Uhl JR, Banerjee R. 2015. Long-term care facilities are reservoirs for antimicrobial-resistant sequence type 131 Escherichia coli. Open Forum Infect Dis 2:ofv011. https://doi.org/10.1093/ofid/ofv011.

30. Johnson JR, Porter S, Thuras P, Castanheira M. 2017. The pandemic H30 subclone of sequence type 131 (ST131) as the leading cause of multidrug-resistant Escherichia coli infections in the United States (2011-2012). Open Forum Infect Dis 4:ofx089. https://doi.org/10.1093/ ofid/ofx089.

31. Tchesnokova V, Rechkina E, Chan D, Haile HG, Larson L, Schroeder DW, Solyanik T, Shibuya S, Hansen KE, Ralston JD, Riddell K, Scholes D, Sokurenko EV. 2019. Pandemic uropathogenic fluoroquinolone-resistant Escherichia coli have enhanced ability to persist in the gut and cause bacteriuria in healthy women. Clin Infect Dis 4:ciz547. https://doi.org/10 .1093/cid/ciz547.

32. Ong SH, Kukkillaya VU, Wilm A, Lay C, Ho EX, Low L, Hibberd ML, Nagarajan N. 2013. Species identification and profiling of complex microbial communities using shotgun Illumina sequencing of 16S rRNA amplicon sequences. PLoS One 8:e60811. https://doi.org/10.1371/journal.pone.0060811.

33. Chen W, Zhang CK, Cheng Y, Zhang S, Zhao H. 2013. A comparison of methods for clustering 16S rRNA sequences into OTUs. PLoS One 8:e70837. https://doi.org/10.1371/journal.pone.0070837.

34. Zolfo M, Tett A, Jousson O, Donati C, Segata N. 2017. MetaMLST: multi-locus clone-level bacterial typing from metagenomic samples. Nucleic Acids Res 45:e7. https://doi.org/10.1093/nar/gkw837.

35. Scholz M, Ward DV, Pasolli E, Tolio T, Zolfo M, Asnicar F, Truong DT, Tett A, Morrow AL, Segata N. 2016. Clone-level microbial epidemiology and population genomics from shotgun metagenomics. Nat Methods 13: 435-438. https://doi.org/10.1038/nmeth.3802.

36. Nayfach S, Rodriguez-Mueller B, Garud N, Pollard KS. 2016. An integrated metagenomics pipeline for strain profiling reveals novel patterns of bacterial transmission and biogeography. Genome Res 26:1612-1625. https://doi.org/10.1101/gr.201863.115.

37. Fischer M, Strauch B, Renard BY. 2017. Abundance estimation and differential testing on strain level in metagenomics data. Bioinformatics 33:i124-i132. https://doi.org/10.1093/bioinformatics/btx237.

38. Weissman SJ, Johnson JR, Tchesnokova V, Billig M, Dykhuizen D, Riddell K, Rogers P, Qin X, Butler-Wu S, Cookson BT, Fang FC, Scholes D, Chattopadhyay S, Sokurenko EV. 2012. High-resolution two-locus clonal typing of extraintestinal pathogenic Escherichia coli. Appl Environ Microbiol 78:1353-1360. https://doi.org/10.1128/AEM.06663-11.

39. Reference deleted.

40. Eckburg PB, Bik EM, Bernstein CN, Purdom E, Dethlefsen L, Sargent M, Gill SR, Nelson KE, Relman DA. 2005. Diversity of the human intestinal microbial flora. Science 308:1635-1638. https://doi.org/10.1126/science .1110591 .

41. Anderson MA, Whitlock JE, Harwood VJ. 2006. Diversity and distribution of Escherichia coli genotypes and antibiotic resistance phenotypes in feces of humans, cattle, and horses. Appl Environ Microbiol 72: 6914-6922. https://doi.org/10.1128/AEM.01029-06.

42. Richter TKS, Hazen TH, Lam D, Coles CL, Seidman JC, You Y, Silbergeld EK, Fraser CM, Rasko DA. 2018. Temporal variability of Escherichia coli diversity in the gastrointestinal tracts of Tanzanian children with and without exposure to antibiotics. mSphere 3:e00558-18. https://doi.org/ 10.1128/mSphere.00558-18.

43. Diard M, Garry L, Selva M, Mosser T, Denamur E, Matic I. 2010. Pathogenicity associated islands in extraintestinal pathogenic Escherichia coli are fitness elements involved in intestinal colonization. J Bacteriol 192: 4885-4893. https://doi.org/10.1128/JB.00804-10.

44. Le Gall T, Clermont O, Gouriou S, Picard B, Nassif X, Denamur E, Tenaillon O. 2007. Extraintestinal virulence is a coincidental by-product of commensalism in B2 phylogenetic group Escherichia coli strains. Mol Biol Evol 24:2373-2384. https://doi.org/10.1093/molbev/msm172.

45. Nielsen KL, Stegger M, Godfrey PA, Feldgarden M, Andersen PS, FrimodtMøller N. 2016. Adaptation of Escherichia coli traversing from the faecal environment to the urinary tract. Int J Med Microbiol 306:595-603. https://doi.org/10.1016/j.ijmm.2016.10.005.

46. Moreno E, Andreu A, Pérez T, Sabaté M, Johnson JR, Prats G. 2006. Relationship between Escherichia coli strains causing urinary tract infection in women and the dominant faecal flora of the same hosts. Epidemiol Infect 134:1015-1023. https://doi.org/10.1017/S0950268806005917.

47. Smati M, Clermont O, Le Gal F, Schichmanoff O, Jauréguy F, Eddi A, Denamur E, Picard B. 2013. Real-time PCR for quantitative analysis of human commensal Escherichia coli populations reveals a high frequency of subdominant phylogroups. Appl Environ Microbiol 79:5005-5012. https://doi.org/10.1128/AEM.01423-13.

48. Philip TLC, Clausen F, Aarestrup M, Lund O. 2018. Rapid and precise alignment of raw reads against redundant databases with KMA. BMC Bioinformatics 19:307. https://doi.org/10.1186/s12859-018-2336-6.

49. Li H. 2018. Minimap2: pairwise alignment for nucleotide sequences. Bioinformatics 34:3094-3100. https://doi.org/10.1093/bioinformatics/ bty 191.

50. Li H, Handsaker B, Wysoker A, Fennell T, Ruan J, Homer N, Marth G, Abecasis G, Durbin R, 1000 Genome Project Data Processing Subgroup. 2009. The sequence alignment/map (SAM) format and SAMtools. Bioinformatics 25:2078-2079. https://doi.org/10.1093/bioinformatics/btp352.

51. Tchesnokova V, Avagyan H, Billig M, Chattopadhyay S, Aprikian P, Chan D, Pseunova J, Rechkina E, Riddell K, Scholes D, Fang FC, Johnson JR, Sokurenko EV. 2016. A novel 7-single nucleotide polymorphism-based clonotyping test allows rapid prediction of antimicrobial susceptibility of extraintestinal Escherichia coli directly from urine specimens. Open Forum Infect Dis 3:ofw002. https://doi.org/10.1093/ofid/ofw002.

52. StataCorp. 2019. Stata statistical software, release 16. StataCorp LLC, College Station, TX.

53. Kumar S, Stecher G, Tamura K. 2016. MEGA7: Molecular Evolutionary Genetics Analysis version 7.0. Mol Biol Evol 33:1870-1874. https://doi .org/10.1093/molbev/msw054.

54. Waskom M, Botvinnik O, O'Kane D, Hobson P, Lukauskas S, Gemperline DC, Augspurger T, Halchenko Y, Cole JB, Warmenhoven J, de Ruiter J, Pye C, Hoyer S, Vanderplas J, Villalba S, Kunter G, Quintero E, Bachant P, Martin M, Meyer K, Miles A, Ram Y, Yarkoni T, Williams ML, Evans C, Fitzgerald C, Fonnesback C, Lee A, Qalieh A. 2017. Seaborn: statistical data visualization. http://seaborn.pydata.org. Retrieved 5 February 2019.

55. Roer L, Tchesnokova V, Allesoe R, Muradova M, Chattopadhyay S, Ahrenfeldt J, Thomsen MCF, Lund O, Hansen F, Hammerum AM, Sokurenko E, Hasman H. 2017. Development of a Web tool for Escherichia coli subtyping based on fimH alleles. J Clin Microbiol 55:2538-2543. https:// doi.org/10.1128/JCM.00737-17. 